\title{
Optical/Multiwavelength Observations of GRB Afterglows
}

\author{
Titus J. Galama ${ }^{1}$ \\ Astronomy, MS 105-24, California Institute of Technology, Pasadena, CA 91125
}

\begin{abstract}
I review $\gamma$-ray burst optical/multiwavelength afterglow observations since 1997, when the first counterparts to GRBs were discovered. I discuss what we have learned from multiwavelength observations of GRB afterglows in relation to the 'standard' fireball plus relativistic blast-wave models. To first order, the 'standard' model describes the afterglow observations well, but a wealth of information can be gathered from the deviations of GRB afterglow observations from this 'standard' model. These deviations provide information on the nature of the progenitor and on the physics of GRB production. In particular, I focus on the possible connection of GRBs to supernovae, on jet and circumstellar wind models, on the early-time afterglow, and on the emission from the reverse shock.
\end{abstract}

\section{INTRODUCTION}

Fireball plus relativistic blast-wave models predict low-energy radiation following GRBs (see e.g., [1]). This radiation has been dubbed the 'afterglow'. The basic model is that of a point explosion: a large amount of energy, $\sim 10^{52-53} \mathrm{ergs}$, is released in a compact region (less than a light millisecond across), which leads to a 'fireball', an optically thick radiation-electron-positron plasma with initial energy much larger than its rest mass that expands ultra-relativistically (see e.g., [2] for an extensive review). The GRB may be due to a series of 'internal shocks' that develop in the relativistic ejecta before they collide with the ambient medium. When the fireball runs into the surrounding medium a 'forward shock' ploughs into the medium and heats it, and a 'reverse shock' does the same to the ejecta. As the forward shock is decelerated by increasing amounts of swept-up material it produces a slowly fading 'afterglow' of X rays, followed by ultraviolet, optical, infrared, millimeter, and radio radiation. As the reverse shock travels through the ejecta it may give rise to a bright optical flash.

Models for the origin of GRBs that (in principle) can provide the required energies, are the neutron star-neutron star (e.g., [3]) and neutron star-black hole

1) The author is supported by the Sherman Fairchild Foundation.

CP526, Gamma-Ray Bursts: $5^{\text {th }}$ Huntsville Symposium, edited by R. M. Kippen, et al. () 2000 American Institute of Physics 1-56396-947-5/00/\$17.00 
mergers [4-6], white dwarf collapse [7], and core collapses of very massive stars ('failed' supernovae or hypernovae $[8,9]$ ).

This review consists of two parts. In the first part, I discuss several confirmations of the relativistic nature of GRB events and discuss the generally good agreement between the 'standard' fireball plus relativistic blast wave model and the observations of GRB afterglows. In the second part, I then proceed to discuss the 'devious' deviations of some GRB afterglows from this standard model, and discuss the wealth of information that we can gather from them. In particular, I discuss what such deviations may tell us about the nature of the progenitor and about the physics of GRB production.

\section{CONFIRMATION OF TIIE RELATIVISTIC BLAST-WAVE MODEL}

\section{A The Forward Shock}

Let us first concentrate on the forward shock and assume slow cooling (the bulk of the electrons do not radiate a significant fraction of their own energy and the evolution is adiabatic); this appears applicable to some observed GRB afterglows at late times $(t>1 \mathrm{hr})$.

The electrons are assumed to be accelerated, in the forward shock, to a powerlaw distribution of electron Lorentz factors, $N\left(\gamma_{\mathrm{e}}\right) \propto \gamma_{\mathrm{e}}^{-p}$, with some minimum Lorentz factor $\gamma_{\mathrm{m}}$. Then, the synchrotron spectrum of such a distribution of electrons is a power law with $F_{\nu} \propto \nu^{1 / 3}$ up to a maximum, $F_{\mathrm{m}}$, at the peak frequency $\nu_{\mathrm{m}}$ (corresponding to the minimum Lorentz factor $\gamma_{\mathrm{m}}$ ). Above $\nu_{\mathrm{m}}$ it is a power law, $F_{\nu} \propto \nu^{-(p-1) / 2}$, up to the cooling frequency, $\nu_{\mathrm{c}}$. Electrons with energies

$\gamma_{\mathrm{e}} m_{\mathrm{e}} c^{2}>\gamma_{\mathrm{c}} m_{\mathrm{e}} c^{2}$, where $\gamma_{\mathrm{c}}$ is the electron Lorentz factor associated with the cooling frequency $\nu_{\mathrm{c}}$, radiate a significant fraction of their energy and thereby cause a spectral transition; above $\nu_{\mathrm{c}}$ we have $F_{\nu} \propto \nu^{-p / 2}$. Synchrotron self absorption causes a steep cutoff of the spectrum at low frequencies, $\nu<\nu_{\mathrm{a}}\left(F_{\nu} \propto \nu^{2}\right.$ if $\left.\nu_{\mathrm{a}}<\nu_{\mathrm{m}}\right)$, where $\nu_{\mathrm{a}}$ is the synchrotron self absorption frequency. Thus, the spectrum consists of four distinct power-law regimes, separated by three break frequencies: (i) the self absorption frequency, $\nu_{\mathrm{a}}$, (ii) the peak frequency, $\nu_{\mathrm{m}}$, and (iii) the cooling frequency, $\nu_{\mathrm{c}}$ (see Fig. 2).

The simplest assumption is that of spherical symmetry and a constant ambient density. For example, if GRBs are the result of the merger of a compact binary system (such as a double neutron star or a neutron star-black hole binary system), then we would expect the fireball to encounter a homogeneous ambient medium. In that case the afterglow can be described by the spectral shape described above combined with the following scalings: $\nu_{\mathrm{m}} \propto t_{\mathrm{obs}}^{-3 / 2}, \nu_{\mathrm{c}} \propto t_{\mathrm{obs}}^{-1 / 2}, \nu_{\mathrm{a}} \propto t_{\mathrm{obs}}^{0}=$ constant, and $F_{\mathrm{m}} \propto t_{\mathrm{obs}}^{0}=$ constant (see [10] and [11] for details). 


\section{The First X-Ray and Optical Counterparts}

Since both the afterglow spectrum and the temporal evolution of the break frequencies $\left(\nu_{\mathrm{a}}, \nu_{\mathrm{m}}\right.$, and $\left.\nu_{\mathrm{c}}\right)$ are power laws in this model, the evolution of the flux is also a power law in time. For example, for $\nu_{\mathrm{m}} \leq \nu \leq \nu_{\mathrm{c}}$, the decay of the flux is $F_{\nu} \propto t_{\mathrm{obs}}^{-3(p-1) / 4}$, and the power-law spectral slope $\alpha$ relates to the spectral slope $\beta$ as $\alpha=-3 / 2 \beta$. A stringent test of the relativistic blast-wave model came with the discovery of the first X-ray [12] and optical [13] counterparts to GRB 970228. Several authors [14-16] showed that to first order the model describes the X-ray and optical afterglow very well (see Fig. 1).

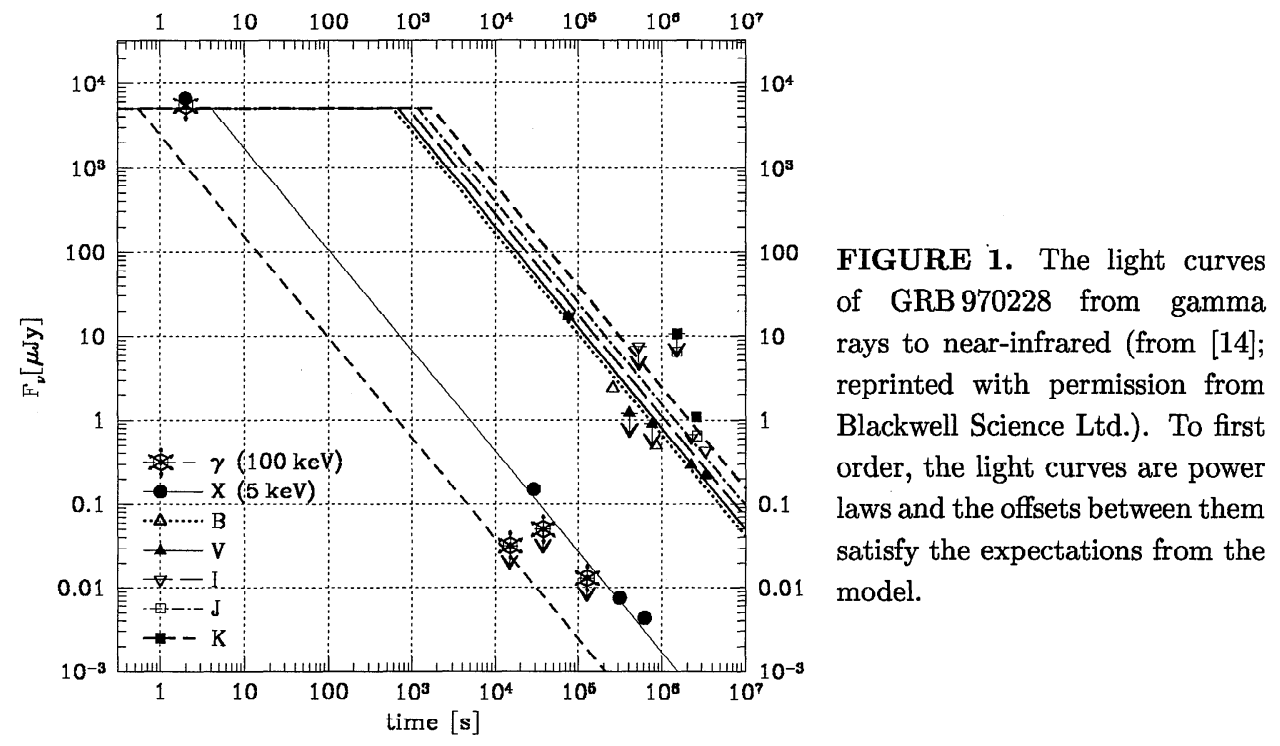

Detection of absorption features in the optical transient (OT) spectrum of GRB 970508 [17] established that this event was at a redshift $z>0.835$, showing that GRBs are located at cosmological distances and are thus extremely powerful events. This was also the first GRB with a radio counterpart [18]. The radio light curves ( 8.5 and $4.9 \mathrm{GHz}$ ) show large variations on time scales of less than a day, but these damp out after one month. This finds a viable explanation in interstellar scintillation (irregular plasma refraction by the interstellar medium between the source and the observer). The damping of the fluctuations can then be understood as the effect of source expansion on the diffractive interstellar scintillation. Thus, a source size of roughly $10^{17} \mathrm{~cm}$ was derived (at 3 weeks), corresponding to a mildly relativistic expansion of the shell [18].

GRB 970508 remains one of the best observed afterglows: the radio afterglow was visible for at least 368 days (and at 2.5 sigma on day 408.6 [19]), and the optical afterglow up to $\sim 450$ days (e.g., [20-23]). In addition, millimeter [24], infrared and X-ray [25] counterparts were detected, and it is the first GRB for 
which a spectral transition in the optical/near-IR range was found [22,26]; this transition is interpreted as the effect of the passage of the cooling frequency through the optical/near-IR passbands. These multiwavelength observations allowed the reconstruction of the broad radio to X-ray spectrum for this GRB [26] (see Fig. 2). It is found that the 'standard' model provides a successful and consistent description of the afterglow observations over nine decades in frequency, ranging in time from the event until several months later [26]. The synchrotron afterglow spectrum of this GRB allows measurement of the electron energy spectrum $p$, the three break frequencies $\left(\nu_{\mathrm{a}}, \nu_{\mathrm{m}}\right.$ and $\left.\nu_{\mathrm{c}}\right)$, and the flux at the peak, $F_{\mathrm{m}}$. For GRB 970508 the redshift, $z$, is also known, and all blast wave parameters could be deduced: the total energy (per unit solid angle) $E=3.5 \times 10^{52} \mathrm{erg}$, the ambient (nucleon) density $n=0.030$, the fraction of the energy in electrons $\epsilon_{\mathrm{e}}=0.12$ and that of the magnetic field $\epsilon_{B}=0.089$ [11]. The numbers themselves are uncertain by an order of magnitude (see e.g., [27]), but the result shows that the 'standard' model fits the expectations very well.

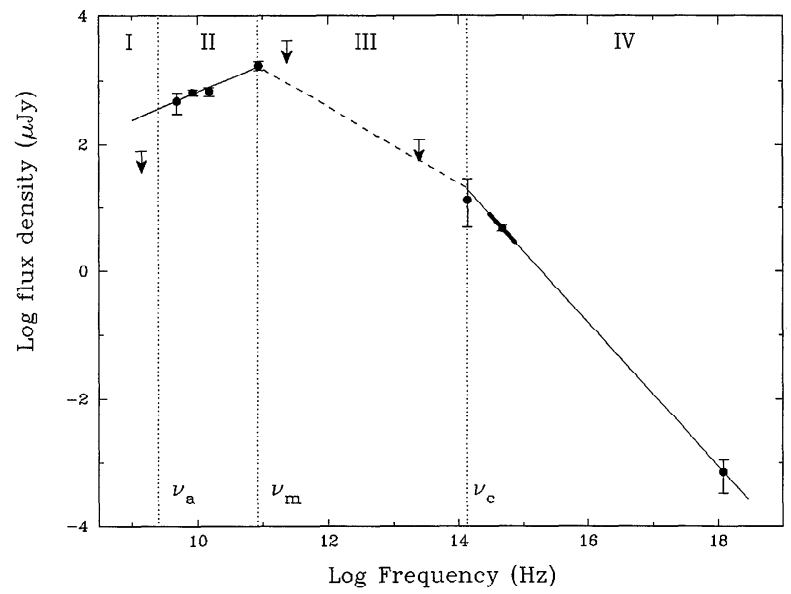

FIGURE 2. The X-ray to radio spectrum of GRB 970508 on May 21.0 UT (12.1 days after the event). The location of the break frequencies $\nu_{\mathrm{a}}, \nu_{\mathrm{m}}$ and $\nu_{\mathrm{c}}$, inferred from transitions in the light curves and from spectra of the afterglow, are indicated (from [26]).

\section{B The Reverse Shock}

The ROTSE telescope obtained its first images only 22 seconds after the start of GRB 990123 (i.e., during the GRB), following a notification received from BATSE aboard the Compton Observatory satellite. The ROTSE observations show that the optical light curve peaked at $\mathrm{m}_{V} \sim 9$ magnitudes some 60 seconds after the event began [28]. After maximum, a fast decay followed for at least 15 minutes. The late-time afterglow observations show a more gradual decline [29-33] (see Fig. 3).

The redshift $z=1.6$, inferred from absorption features in the OT spectrum, implies that the optical flash would have been as bright as the full moon had the GRB occurred in the nearby galaxy M31 (Andromeda). If one assumes that the emission detected by ROTSE comes from a non-relativistic source of size $c t$, 


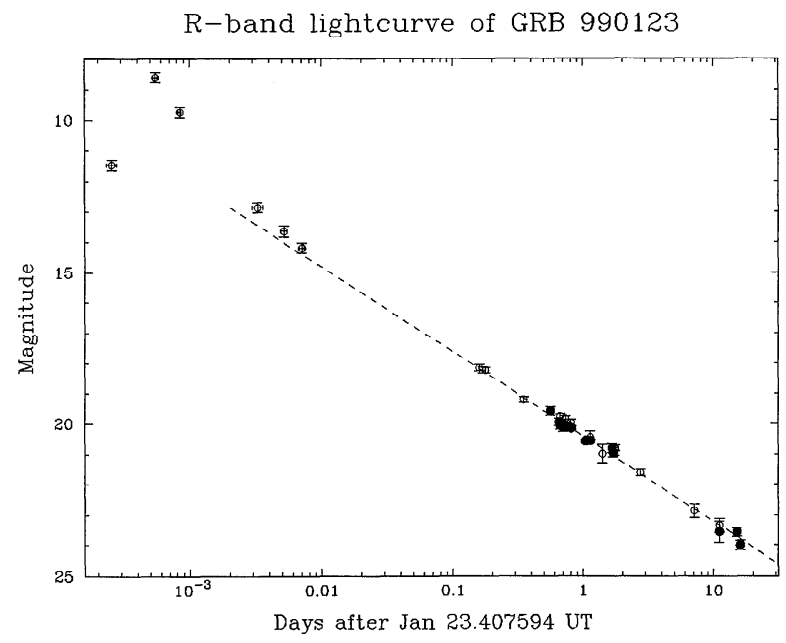

FIGURE 3. R-band light curve of the afterglow of GRB 990123. The R.OTSE data show that the optical light curve peaked at $\mathrm{m}_{V} \sim 9$ [28]. The dashed line indicates a power-law fit to the light curve (for $t>0.1$ days), which has exponent $-1.12 \pm 0.03$ (Reprinted by permission from Nature [29] copyright (1999) Macmillan Magazines Ltd.).

then the observed brightness temperature $T_{b} \gtrsim 10^{17} \mathrm{~K}$ of the optical flash exceeds the Compton limit of $10^{12} \mathrm{~K}$, confirming the highly relativistic nature of the GRB source [29].

The RO'TSE observations show that the prompt optical and $\gamma$-ray light curves do not track each other [28]. In addition, detailed comparison of the prompt optical emission with the BATSE spectra of GRB 990123 (at three epochs for which both optical and gamma-ray information is available) shows that the ROTSE emission is not a simple extrapolation of the GRB spectrum to much lower energies [29,34].

Emission from the reverse shock is predicted to peak near the optical waveband during or just after the GRB $[1,35]$. The observed properties of GRB 990123 appear to fit this model quite well $[29,33,36]$. If this interpretation is correct, GRB 990123 would be the first burst in which all three emitting regions have been seen: internal shocks causing the GRB, the reverse shock causing the prompt optical flash, and the forward shock causing the afterglow. The emissions thus arise from three different emitting regions, explaining the lack of correlation between the GRB, the prompt optical and the late-time optical emission [29] (but see [37]).

\section{DEVIATIONS}

As discussed above, the 'standard' model explains the multiwavelength obser-

vations of GRBB afterglows very well. Now that we have a basic understanding of GRB afterglows it is interesting to consider what we can learn (and what we have learned in the past year) from the observational departures from the 'standard' model. 

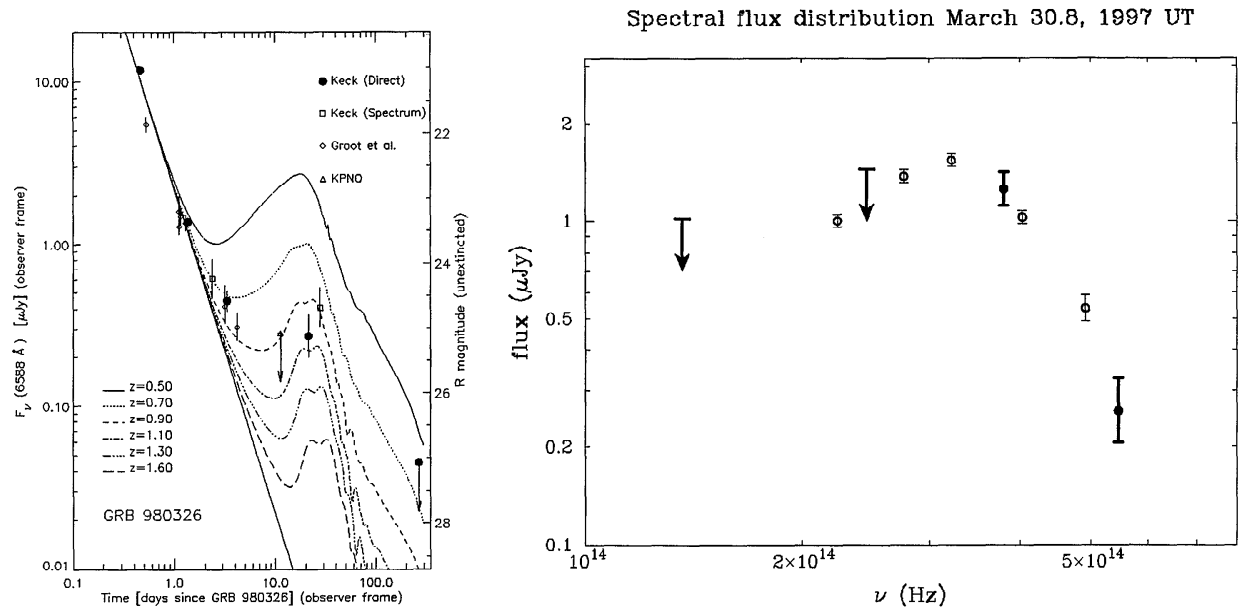

FIGURE 4. Left: R-band light curve of GRB 980326 and the sum of an initial power-law decay plus Ic supernova light curve for redshifts ranging from $z=0.50$ to $z=1.60$ (Reprinted by permission from Nature [40] copyright (1999) Macmillan Magazines Ltd.). Right: The broad-band spectrum of the OT of GRB 970228 at March 30.8, 1997 UT (• and upper-limit arrow). Also shown is the spectral flux distribution of SN $1998 \mathrm{bw}$ (०) redshifted to the redshift of GRB 970228 $(z=0.695)$. The similarity of the spectral flux distributions is remarkable (from [43]).

\section{A The GRB/Supernova Connection}

A direct consequence of the collapsar model is that GRBs are expected to be accompanied by supernovae ( $\mathrm{SNe}$ ).

The first evidence for a possible GRB/SN connection was provided by the discovery by Galama et al. [38] of SN 1998bw in the error box of GRB 980425. The temporal and spatial coincidence of SN $1998 \mathrm{bw}$ with GRB 980425 suggest that the two phenomena are related $[38,39]$. GRB 980425 is most certainly not a typical GRB: the redshift of SN $1998 \mathrm{bw}$ is 0.0085 and the corresponding $\gamma$-ray peak luminosity of GRB 980425 and its total $\gamma$-ray energy budget are about a factor of $\sim$ $10^{5}$ smaller than those of 'normal' GRBs. Such SN-GRBs may well be the most frequently occurring GRBs in the Universe.

Bloom et al. [40] realized that the late-time red spectrum and the late-time rebrightnening of the light curve of GRB 980326 are possible evidence that at late times the emission is dominated by an underlying supernova. The authors find that a template supernova light curve, provided by the well-studied type $\mathrm{I}_{b / c} \mathrm{SN} 1998 \mathrm{bw}$ provides an adequate description of the observations (see Fig. 4).

In fact, the behavior of GRB 970228 already showed first indications that the standard model was not sufficient to describe the observations in detail [41]. The early-time decay of the optical emission is faster than that at later times and, as the source faded, it showed an unexpected reddening [41]. Indeed, Galama et al. 
[41] conclude that although the initial behavior is in agreement with the 'standard' model, the subsequent behavior is harder to explain. It was not until Bloom et al. [40] discussed evidence for a supernova-like emission accompanying GRB 980326 that the behavior of GRB 970228 was better understood. Also for GRB 970228 the late-time light curve and reddening of the transient can be well explained by an initial power-law decay modified at late times by SN 1998bw-like emission $[42,43]$ (see Fig. 4).

The relation between distant GRBs like GRB 980326 and GRB980425/SN $1998 \mathrm{bw}$ is unclear. Is SN 1998bw a different phenomenon or a more local and lower energy equivalent? Are all afterglows consistent with such a phenomenon? The answer to the latter question requires detailed analysis of existing data on GRB afterglows, but more convincing evidence may be provided by future observations of GRB afterglows around the time of the SN emission maximum.

\section{B Collimated Outflow (Jet) and/or Circumstellar Wind Model}

If, as suggested by the evidence for a GRB/SN connection (see §III A), at least some GRBs are produced by the core collapse of massive stars to black holes, then the circumburst environment will have been influenced by the strong wind of the massive progenitor star. For a constant wind speed, the circumstellar density falls as $n \propto r^{-2}$, where $r$ is the radial distance. In this so called circumstellar wind model, the afterglow can be described by the same synchrotron spectral shape (see $\S I \mathrm{~A}$ ), but with different scalings for the break frequencies and the peak flux: $\nu_{\mathrm{m}} \propto t_{\mathrm{obs}}^{-3 / 2}, \nu_{\mathrm{c}} \propto t_{\mathrm{obs}}^{+1 / 2}, \nu_{\mathrm{a}} \propto t_{\mathrm{obs}}^{-3 / 5}$, and $F_{\mathrm{m}} \propto t_{\mathrm{obs}}^{-1 / 2}$ (see $[45,46]$ for details).

Due to relativistic beaming, only a small portion of the emitting surface with opening angle $1 / \gamma$ is visible. As the fireball evolves $\gamma$ decreases and the beaming angle will eventually exceed the angular size of the collimated outflow (the size of the jet). In this jet model, we then expect to see an increase in the decay rate. Slightly later, the jet begins a lateral expansion, which causes a further steepening of the light curve. In this case the scalings for the break frequencies are: $\nu_{\mathrm{m}} \propto t_{\mathrm{obs}}^{-2}$, $\nu_{\mathrm{c}} \propto t_{\mathrm{obs}}^{0}=$ constant, $\nu_{\mathrm{a}} \propto t_{\mathrm{obs}}^{-1 / 5}$, and $F_{\mathrm{m}} \propto t_{\mathrm{obs}}^{-1}$ (see for details [47-49]). At late times, when the evolution is dominated by the spreading of the jet, the decay is as fast as $\mathrm{F}_{\nu}(t) \propto t^{-p} \sim t^{-2.2}$, where $p$ is the power-law index of the electron energy spectrum.

Non 'standard' behavior: The optical and X-ray light curves of GRB 970508 show a maximum that is reached around 1 day and is followed by characteristic power-law decaying light curves. The onset of the X-ray flare roughly coincides with that of the optical bump [25]. This behavior is not yet well understood. Panaitescu et al. [44] have tested several possible models to explain the flare: (i) by continued energy injection from the central source, (ii) by ejecta with a range of Lorentz factors, (iii) as the effect of a jet that is observed slightly off-center, and (iv) by the encounter of a shell of dense ambient material. 
The afterglow peak flux $\mathrm{F}_{\mathrm{m}}$ of GRB 970508 decays with time; it is $\sim 1700 \mu \mathrm{Jy}$ at $86 \mathrm{GHz}$ at $\sim 12$ days, while only $\sim 700 \mu \mathrm{Jy}$ at $8.5 \mathrm{GHz}$ at $\sim 60$ days $[18,24,26]$. Also, the self-absorption frequency $\nu_{\mathrm{a}}$ evolves to lower frequencies. However, in the 'standard' model the peak flux and the self-absorption frequency would remain constant in time. Again, these features have several possible explanations: (i) the effect of collimated outflow, (ii) the effect of a circumstellar wind, or (iii) the transition from an ultra-relativistic to a non-relativistic evolution [19]. Note however, that the 'standard' model and the circumstellar wind model predict a distinctively different evolution of the cooling break $\nu_{\mathrm{c}}$; the observed evolution for GRB 970508 fits the 'standard' model well and is hard to reconcile with the wind model.

Fast decaying afterglows: GRB 980326 was the first example of a rapidly decaying afterglow [50]. Unfortunately, no attempt was made to observe the X-ray afterglow, and the optical spectral information is only sparse. It was not until GRB 980519 that it was decisively found that the rapidly decaying afterglow could not be understood in the terms of the 'standard' model; the relation between the spectral slope and the temporal decay is not as expected from the 'standard' model. The observations can either be explained by a jet $[48,51]$ or by a circumstellar wind model [45]. Radio observations of GRB 980519 are well described by a wind model, but cannot decisively reject the jet model [52]. The reason that it is hard to distinguish the different models is because of the absence of high quality data; afterglows are faint. Future radio observations at early and late times may allow to decisively distinguish the models.

Perhaps the actual light-curve transition (from a regular to a fast decay caused by 'seeing' the edge of the jet) has been observed in the optical afterglow of GRB 990123 [30-32]. However, no evidence for such an increase of the decay rate was found in near-infrared K-band observations [30]. A similar transition was better sampled in afterglow data of GRB 990510; optical observations of GRB 990510, show a clear steepening of the rate of decay of the light between $\sim 3$ hours and several days $[53,54]$ to roughly $\mathrm{F}_{\nu}(t) \sim t^{-2.2}$. Together with radio observations, which also reveal a transition, it is found that the transition is very much frequencyindependent; this virtually excludes explanations in terms of the passage of the cooling frequency, but is what is expected in case of beaming [53]. Harrison et al. [53] derive a jet opening angle of $\theta=0.08$, which for this burst would reduce the total energy in $\gamma$ rays to $\sim 10^{51} \mathrm{erg}$.

\section{The Early Afterglow and the Reverse Shock}

The radio observations of GRB 990123 show a brief flare at one day after the event $[29,56]$. Such radio behavior is unique, both for its early appearance as well as its rapid decline. The flare has been suggested to be due to the reverse shock [33,52]. However, understanding the full evolution still requires interpretation in terms of the forward shock and a jet in addition to the reverse shock. An alternative interpretation in terms of emission by the forward shock only is also consistent 
with the observations [29]. This interpretation is also not without problems; the spectrum is required to be relatively flat around the maximum. In this interpretation the energy density of the magnetic field is very low $\epsilon_{B}<10^{-6}$, similar to what is derived for GRB 980703 [55]. The differences in afterglow behavior may thus reflect variations in the magnetic-field strength in the forward shock [29]. Other possibilities have been put forward: an explanation in terms of the forward shock and a jet [57] and an explanation in terms of the forward shock and a dense ambient medium [58]. Interestingly, observations of the light curve at times between 15 min and several hours could distinguish between some of the models; this is the region of transition from early times, where the emission is believed to be due to the reverse shock, to late times where the emission of the forward shock is dominant. The imminent launch of HETE-2 will provide the unique possibility to study this time window, by providing accurate localizations to the community within minutes after the events.

\section{CONCLUSIONS}

Although the 'standard' model describes the afterglow observations well, a wealth of information is provided by the deviations of GRB afterglows from the 'standard' model; in particular, by the possible connection of GRBs to supernovae, by possible evidence for collimated outflow and circumstellar winds, by the early-time afterglow and by the emission from the reverse shock.

\section{REFERENCES}

1. Mészáros, P. and Rees, M.J., ApJ 476, 232 (1997).

2. Piran, T., Pllysics Report, in press, astro-ph//9810256 (1999).

3. Eichler, D., et al., Nature 340, 126 (1989).

4. Mochkovitch, R., et al., Nature 361, 236 (1993).

5. Lattimer, J.M. and Schramm, D.N., ApJL 192, L145 (1974).

6. Narayan, R., Paczyński, B. and Piran, T., ApJL 395, L83 (1992).

7. Usov, V.V., Nature 357, 472 (1992).

8. Woosley, S.E., ApJ 405, 273 (1993).

9. Paczyński, B., ApJL 494, L45 (1998).

10. Sari, R., Piran, T. and Narayan, R., ApJL 497, L17 (1998).

11. Wijers, R.A.M.J. and Galama, T.J., $A p J$ 523, 177 (1999).

12. Costa, E., et al., Nature 387, 783 (1997).

13. Van Paradijs, J., et al., Nature 386, 686 (1997).

14. Wijers, R.A.M.J., Rees, M.J. and Mészáros, P., MNRAS 288, L51 (1997).

15. Reichart, D.E., ApJL 485, L57 (1997).

16. Waxman, E., ApJL 485, L5 (1997).

17. Metzger, M.R., et al., Nature 387, 879 (1997).

18. Frail, D.A., et al., Nature 389, 261 (1997). 
19. Frail, D.A., Waxman, E. and Kulkarni, S.R., ApJ, in press, astro-ph/9910319 (2000).

20. Fruchter, A., et al., ApJL, submitted, astro-ph/9902236 (1999).

21. Bloom, J.S., et al., ApJL 507, L25 (1998).

22. Galama, T.J., et al., ApJL 497, L13 (1998).

23. Castro-Tirado, A., et al., Science 279, 1011 (1998).

24. Bremer, M., et al., $A \& A$ 332, L13 (1998).

25. Piro, L., et al., $A \& A$ 331, L41 (1998).

26. Galama, T.J., et al., ApJL 501, L97 (1998).

27. Granot, J., Piran, T. and Sari, R., ApJL 527, 236 (1999).

28. Akerlof, C., et al., Nature 398, 400 (1999).

29. Galama, T.J., et al., Nature 398, 394 (1999).

30. Kulkarni, S.R., et al., Nature 398, 389 (1999).

31. Castro-Tirado, A., et al., Science 283, 2069 (1999).

32. Fruchter, A.S., et al., ApJL 519, L13 (1999).

33. Sari, R. and Piran, T., ApJL 517, L109 (1999).

34. Briggs, M.S., et al., $A p J$ 524, 82 (1999).

35. Sari, R. and Piran, T., ApJL 520, 641 (1999).

36. Mészáros, P. and Rees, M. J., MNRAS 306, L39 (1999).

37. Liang, E.P., et al., ApJL 519, L21 (1999).

38. Galarna, T.J., et al., Nature 395, 670 (1998).

39. Kulkarni, S.R., et al., Nature 395, 663 (1998).

40. Bloom, J.S., et al., Nature 401, 453 (1999).

41. Galama, T.J., et al., Nature 387, 479 (1999).

42. Reichart, D.E., ApJL 521, L111 (1999).

43. Galama, T.J., et al., ApJ, submitted, astro-ph/9907264 (1999).

44. Panaitescu, A., Mészáros, P., and M. J. Rees, M.J., ApJ 503, 314 (1998).

45. Chevalier, R.A. and Li, Z., ApJL 520, L29 (1999).

46. Chevalier, R.A. and Li, Z., ApJ, submitted, astro-ph/9908272 (1999).

47. Rhoads, J.E., ApJ 525, 737 (1999).

48. Sari, R., Piran, T. and Halpern, J.P., ApJL 519, L17 (1999).

49. Panaitescu, A. and Mészáros, P., ApJ 526, 707 (1999).

50. Groot, P.J., et al., ApJL 502, L123 (1998).

51. Halpern, J.P., $A p J L$ 517, L105 (1999).

52. Frail, D.A., et al. ApJ, submitted, astro-ph/9910060 (1999).

53. Harrison, F.A., et al., ApJL 523, L121 (1999).

54. Stanek, K.Z., et al., ApJL 522, L39 (1999).

55. Vreeswijk, P.M., et al., ApJL 523, 171 (1999).

56. Vreeswijk, P.M., et al., ApJL 522, L97 (1999).

57. Wang, X.Y., Dai, Z.G. and Lu, T., MNRAS, submitted, astro-ph/9906062 (2000).

58. Dai, Z.G. and Lu, T., ApJL 519, L155 (1999). 